\title{
AVALIAÇÃO CATALÍTICA DE MOLIBDÊNIO SUPORTADO EM NIÓBIA NA DESIDROGENAÇÃO OXIDATIVA DO PROPANO
}

\author{
B. D. COSTA ${ }^{1}$, J.A.J. RODRIGUES ${ }^{2}$ e G. G. CORTEZ ${ }^{1}$ \\ ${ }^{1}$ Escola de Engenharia de Lorena - USP - Departamento de Engenharia Química \\ ${ }^{2}$ Laboratório de Combustão e Propulsão - Instituto Nacional de Pesquisas Espaciais \\ E-mail para contato: cortez@dequi.eel.usp.br
}

\begin{abstract}
RESUMO - A demanda no consumo energético nos atuais métodos de produção de alcenos via desidrogenação oxidativa $(\mathrm{ODH})$ de alcanos vem aumentando nas últimas décadas. No processo de ODH de propano, muitos sistemas catalíticos estudados foram baseados em molibdênio a temperaturas de 450 a $550^{\circ} \mathrm{C}$. Estudos mostraram que a nióbia pura é um catalisador muito seletivo para a ODH de propano. Neste projeto, o objetivo foi avaliar a atividade catalítica do molibdênio suportado em nióbia preparada por método hidrotérmico. A impregnação do molibdênio foi realizada via impregnação úmida com excesso de solvente, totalizando três catalisadores, os quais foram caracterizados pelas técnicas de volumetria de $\mathrm{N}_{2}$, RTP, DRX e pela reação de ODH de propano. Através do BET concluiu-se que a adição de molibdênio promoveu uma diminuição nos valores de área específica e volume de poros. Pela reação de $\mathrm{ODH}$ notou-se que a adição de molibdênio ao suporte não provoca mudanças significativas na conversão do propano e no rendimento de propeno.
\end{abstract}

PALAVRAS-CHAVE: Molibdênio; Nióbio; ODH; Propano; Catalisadores Mo-Nb.

\section{INTRODUÇÃO}

A demanda de poliolefinas vem crescendo consideravelmente em grande parte dos países ricos e em desenvolvimento, o que torna necessária uma maior produção de eteno e propeno, que são matérias primas para produção de diversos produtos industriais. A desidrogenação oxidativa (ODH) de alcanos leves é uma rota alternativa para a produção de alcenos a partir de alcanos devido ao grande consumo energético, encontrados na desidrogenação catalítica e no craqueamento a vapor, para a produção de olefinas (Mamedov e Cortes-Corberan, 1995). Os esforços atuais estão concentrados na concepção de catalisadores mais eficientes, os quais produzem propeno com rendimentos mais elevados em maiores conversões.

As aplicações catalíticas do óxido de nióbio como suporte, promotor ou um sólido com propriedades ácidas, têm sido reportadas nos últimos anos (Ziolek, 2003). Óxidos metálicos do grupo $\mathrm{V}$ suportados têm uma grande variedade de aplicações catalíticas e têm sido extensivamente investigados (Cortez et. al., 2003). Para a ODH de propano, muitos sistemas estudados foram baseados em molibdênio (Heracleous et. al., 2005) a temperaturas de 450 a $550^{\circ} \mathrm{C}$. O estudo de Yuan et. al., (2005), mostrou que catalisadores mesoporosos Nb-Mo-O, contendo espécies moleculares de 


\section{9 a 22 de outubro de 2014 \\ Florianópolis/SC}

$\mathrm{MoO}_{\mathrm{x}}$ dispersas, apresentaram altas atividades na $\mathrm{ODH}$ de propano do que catalisadores suportados $\mathrm{MoO}_{\mathrm{x}} / \mathrm{Nb}_{2} \mathrm{O}_{5}$, nos quais o óxido de molibdênio estava presente como espécies moleculares dispersas na forma de $\mathrm{MoO}_{\mathrm{x}}$ e cristais de $\mathrm{MoO}_{3}$.

Neste contexto, o presente trabalho tem como objetivo principal avaliar as características texturais de catalisadores de molibdênio suportado em nióbia, preparada a partir do método hidrotérmico, e a atividade catalítica na ODH de propano. A caracterização do suporte e dos catalisadores foi realizada através das técnicas de volumetria de nitrogênio, difratometria de raios $\mathrm{X}$ e redução à temperatura programada. A atividade catalítica foi analisada através da reação de desidrogenação oxidativa do propano.

\section{EXPERIMENTAL}

\subsection{SÍNTESE DOS CATALISADORES}

Neste trabalho, foram sintetizados três catalisadores, identificados como $1 \mathrm{Mo} / \mathrm{Nb}, 2 \mathrm{Mo} / \mathrm{Nb}$ e $3 \mathrm{Mo} / \mathrm{Nb}$. O suporte de óxido de nióbio $\left(\mathrm{Nb}_{2} \mathrm{O}_{5}\right)$, ou nióbia, foi preparado a partir do ácido nióbico $\mathrm{HY}$ 340, o qual foi fornecido pela Companhia Brasileira de Metalurgia e Mineração (CBMM). Para a síntese do suporte, o ácido nióbico foi submetido a um tratamento hidrotérmico.

A impregnação do molibdênio no suporte foi realizada por impregnação úmida com excesso de solvente (água). Na preparação dos catalisadores $x \mathrm{MoO}_{3} / \mathrm{Nb}_{2} \mathrm{O}_{5}$, utilizou-se uma solução aquosa de $\left(\mathrm{NH}_{4}\right)_{6} \mathrm{Mo}_{7} \mathrm{O}_{24} \cdot 4 \mathrm{H}_{2} \mathrm{O}$. A massa desse sal foi calculada de forma que a quantidade de molibdênio por área do suporte, ao final dos tratamentos térmicos subsequentes, seja de $x=1,2$ e 3 átomos $/ \mathrm{nm}^{2}$ sobre o suporte de óxido de nióbio calcinado a $500^{\circ} \mathrm{C}$. Após a dissolução do sal no balão do rotaevaporador, foi adicionada a massa do óxido de nióbio previamente calculada para a impregnação. Após um período de uma hora, o solvente foi retirado a $70^{\circ} \mathrm{C}$ sob vácuo. A etapa seguinte à impregnação foi a calcinação, na qual os catalisadores foram secos a $110^{\circ} \mathrm{C}$ por 24 horas em estufa e, depois, calcinados a $500^{\circ} \mathrm{C}$ por 4 horas em mufla.

\subsection{CARACTERIZAÇÃO FISICO-QUÍMICA}

As medidas de áreas específicas, volume de poros (método BET) e distribuição de volume de poros (método BJH) foram determinadas a partir das isotermas de adsorção-dessorção de $\mathrm{N}_{2}$ a $196^{\circ} \mathrm{C}$, utilizando-se um equipamento da marca BEL Japan, INC., modelo BelSorp mini II. As amostras foram previamente tratadas a $300^{\circ} \mathrm{C}$, por $1 \mathrm{~h}$, em nitrogênio.

As análises por difratometria de raios $\mathrm{X}$ (DRX) foram realizadas empregando-se o método do pó, utilizando-se um equipamento da marca Panalytical, modelo Enpyrean, radiação CuK $\alpha$ obtida em $40 \mathrm{kV}$. Os difratogramas de raios $\mathrm{X}$ foram obtidos com ângulo de varredura de $2 \theta$ variando de 5 a $90^{\circ}$. As fases cristalinas foram identificadas com auxílio da biblioteca do JCPDS - Joint Committee of Powder Diffraction Standards (PCPDFWIN, 2002).

O perfil de redução das espécies redutíveis presentes nos catalisadores foi determinado pela 
técnica de redução à temperatura programada (RTP), utilizando-se um equipamento da marca Quantachrome, modelo Chembet-3000, equipado com um detector de condutividade térmica. As amostras $(15 \mathrm{mg})$ foram secas in situ a $200^{\circ} \mathrm{C}$ por $1 \mathrm{~h}$ sob fluxo de hélio. Posteriormente, os sólidos foram resfriados à temperatura ambiente e, em seguida, reduzidos até $700^{\circ} \mathrm{C}\left(10^{\circ} \mathrm{Cmin}^{-1}\right)$ com uma mistura de $10 \% \mathrm{H}_{2} / \mathrm{N}_{2}\left(30 \mathrm{mLmin}^{-1}\right)$.

Os catalisadores $(100 \mathrm{mg}$ ) foram avaliados na reação de desidrogenação oxidativa (ODH) do propano entre as temperaturas de reação de 300 a $520^{\circ} \mathrm{C}$. Foram adicionados $200 \mathrm{mg}$ de óxido misto de $\mathrm{Mo}-\mathrm{Nb}$ (inerte à $\mathrm{ODH}$ ) e misturados ao catalisador no reator com o objetivo de melhorar a difusão das moléculas de propano sobre a superfície do catalisador. A mistura de gases alimentada ao reator foi de $\mathrm{O}_{2} / \mathrm{C}_{3} \mathrm{H}_{8} / \mathrm{He}(3 / 2 / 6$ molar) com um fluxo total de $45 \mathrm{~mL} / \mathrm{min}$. O propano e os produtos não convertidos foram analisados por um cromatógrafo gasoso em linha, modelo Varian 450, equipado com um TCD, utilizando-se uma coluna Cromossorb 107 e uma peneira molecular 4A. Os produtos principais de reação foram propeno, água, e $\mathrm{CO}_{2}$. A conversão do propano foi calculada a partir de um balanço de quantidade de carbono, assumindo como sendo constante o balanço de carbono presente na saída do reator e no fluxo de alimentação do reagente. Baseado nas concentrações de entrada e saída e assumindo as condições de um reator diferencial, a conversão total $\left(\mathrm{X}_{\mathrm{A}}\right)$, a seletividade $\left(S_{p}\right)$ e o rendimento aos produtos $\left(R_{p}\right)$ foram calculados através das seguintes equações:

$$
X_{A}(\%)=\left(\frac{n_{R}}{n_{R 0}}\right) \cdot 100 \quad S_{p}(\%)=\left(\frac{n_{P}}{n_{I}}\right)\left(\frac{N_{C P}}{N_{C R}}\right) \cdot 100 \quad R_{p}(\%)=X_{A} \cdot S_{p}
$$

onde $\mathrm{n}_{\mathrm{R}}, \mathrm{n}_{\mathrm{R} 0}$ são os números de moles do reagente consumidos e alimentados, respectivamente; $\mathrm{n}_{\mathrm{P}}$ o número de moles dos produtos formados; e $\mathrm{N}_{\mathrm{CP}}$ e $\mathrm{N}_{\mathrm{CR}}$ são os números de átomos de carbono presentes nos produtos formados e no reagente alimentado, respectivamente. A frequência de rotação (TOF - Turn-over-frequency) expressa a atividade catalítica como o número de moles de propano reagidos por unidade de molibdênio por segundo, assumindo que todo o molibdênio seja ativo. O TOF é calculado a partir da seguinte equação:

$$
\mathrm{TOF}=\frac{4,386 \cdot 10^{-3} \cdot \% \text { conversão do propano }}{\% \mathrm{MoO}_{3}}
$$

\section{RESULTADOS E DISCUSSÃO}

As áreas específicas $(\mathrm{Sg})$, volume total de poros $(\mathrm{Vp})$ e diâmetro médio dos poros (Dp) do suporte e dos catalisadores $x \mathrm{Mo} / \mathrm{Nb}$ são apresentados na Tabela 1.

A adição crescente de molibdênio sobre o suporte $\mathrm{Nb}_{2} \mathrm{O}_{5}$ diminui os valores da área específica e do volume de poros, efeito este provocado pela possível obstrução dos poros de menor diâmetro. Entretanto, esse aumento crescente de Mo no interior dos poros do suporte causa um colapso durante o tratamento térmico, resultando em um aumento nos valores dos diâmetros médios dos poros nos catalisadores, que se mantêm praticamente constantes apesar da adição crescente de Mo ao suporte. 
Tabela 1 - Propriedades texturais das amostras calcinadas.

\begin{tabular}{ccccc}
\hline Amostra & $\% \mathrm{Mo}(\mathrm{p} / \mathrm{p})$ & $\mathrm{Sg}\left(\mathrm{m}^{2} / \mathrm{g}\right)$ & $\mathrm{Vp}\left(\mathrm{cm}^{3} / \mathrm{g}\right)$ & $\mathrm{Dp}(\mathrm{nm})$ \\
\hline $\mathrm{Nb}_{2} \mathrm{O}_{5}$ & 0 & 74,0 & 0,19 & 10,0 \\
$1 \mathrm{Mo} / \mathrm{Nb}$ & 1,19 & 23,0 & 0,13 & 22,0 \\
$2 \mathrm{Mo} / \mathrm{Nb}$ & 2,15 & 15,0 & 0,08 & 23,0 \\
$3 \mathrm{Mo} / \mathrm{Nb}$ & 3,19 & 13,0 & 0,07 & 23,0 \\
\hline
\end{tabular}

As isotermas de adsorção-dessorção de $\mathrm{N}_{2}$ a $-196^{\circ} \mathrm{C}$ e as curvas de distribuição de poros do suporte e dos catalisadores são apresentadas na Figura 1. Observa-se a predominância de isotermas do tipo IV, que segundo a nomenclatura da IUPAC, são típicas de materiais mesoporosos (Roqueirol et al., 1994). Nota-se também, que a adição do molibdênio sobre o suporte não modificou o perfil das isotermas. Observa-se um "loop" de histerese do tipo $\mathrm{H} 3$ a 0,40< $\mathrm{P} / \mathrm{P}_{0}<0,9$ nas isotermas entre as etapas de adsorção-dessorção associado a condensação capilar em poros na forma de fendas.

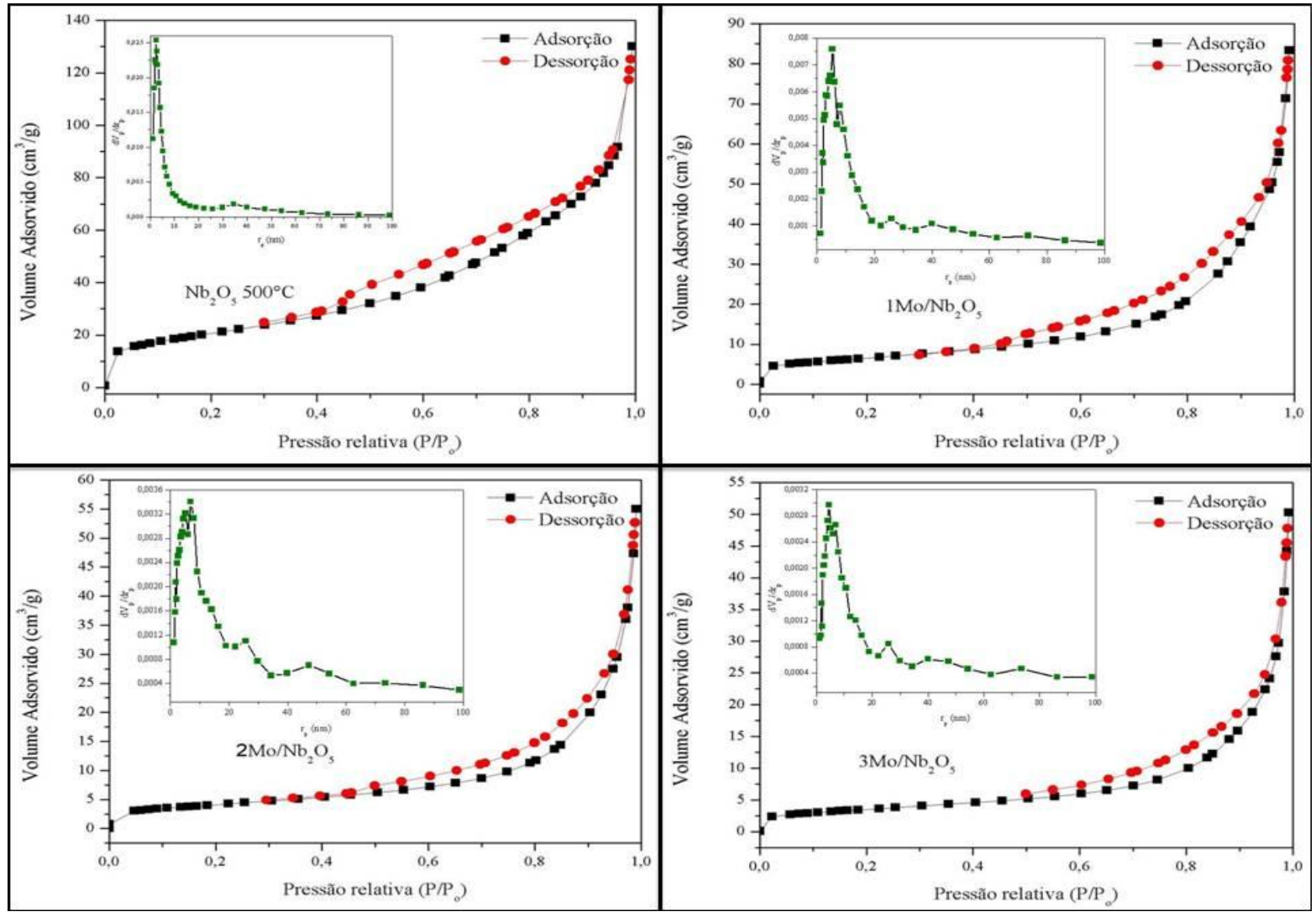

Figura 1 - Isotermas de adsorção-dessorção de $\mathrm{N}_{2}$ e distribuição de volume de poros do suporte e dos catalisadores $x \mathrm{Mo} / \mathrm{Nb}$. 
O difratograma de raios-X do suporte é apresentado na Figura 2. A nióbia preparada pelo método hidrotérmico e calcinada a $500^{\circ} \mathrm{C}$ apresenta estrutura amorfa, tendo início um processo de formação de planos cristalográficos ordenados no pico mais intenso em $2 \theta=22,69^{\circ}$ que é característico da fase TT ou T do óxido de nióbio, resultado obtido também em estudos de Chary et. al., (2003). A adição de $\mathrm{MoO}_{3}$ não provoca mudanças no perfil do DRX, o que indica que os óxidos estão presentes em um estado amorfo, ou altamente dispersos sobre o suporte, porém não se descarta a possibilidade de haver cristalitos desses óxidos com dimensões inferiores a 4nm, uma vez que esse é o limite inferior de detecção de estruturas cristalinas pela técnica de DRX (Chary et. al., 2003).

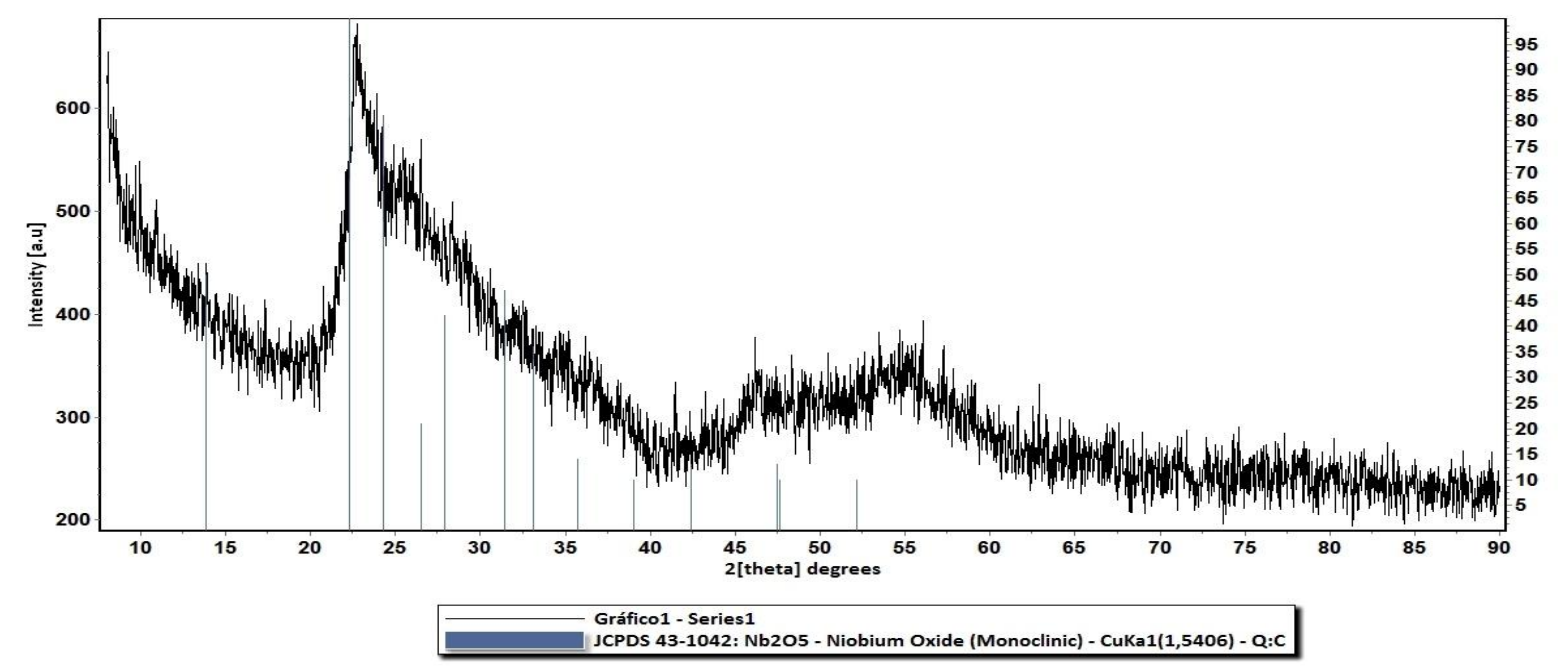

Figura 2 - Difratometria de Raios-X do suporte $\mathrm{Nb}_{2} \mathrm{O}_{5}$ calcinado à $500^{\circ} \mathrm{C}$

As análises de redução a temperatura programada (RTP) dos catalisadores são apresentadas na Figura 3. $\mathrm{O}$ catalisador $1 \mathrm{Mo} / \mathrm{Nb}$ apresentou uma região de redução com maior intensidade em $595^{\circ} \mathrm{C}$. $\mathrm{O}$ catalisador $2 \mathrm{Mo} / \mathrm{Nb}$ apresentou um pico em $627^{\circ} \mathrm{C}$ e um ombro a $535^{\circ} \mathrm{C}$ e o $3 \mathrm{Mo} / \mathrm{Nb}$ um pico de redução a $635^{\circ} \mathrm{C}$ e um ombro em torno de $579^{\circ} \mathrm{C}$. As intensidades dos picos de redução aumentam nos catalisadores conforme aumento da quantidade de molibdênio no suporte, além disso, ocorre um deslocamento do pico de maior intensidade. A interação do molibdênio com o nióbio pode ter formado um óxido misto, já que o nióbio mássico reduz a temperaturas elevadas $\left(>800^{\circ} \mathrm{C}\right)$ e, de acordo com Arnoldy et.al., (1985), a redução do molibdênio ocorre essencialmente em duas etapas, cujos picos foram atribuídos à seguinte sequência de redução:

$$
\begin{gathered}
\mathrm{MoO}_{3} \rightarrow \mathrm{MoO}_{2} \text { a } 765^{\circ} \mathrm{C} \\
\mathrm{MoO}_{2} \rightarrow \text { Mo a } 997^{\circ} \mathrm{C}
\end{gathered}
$$

$\mathrm{Na}$ primeira etapa, a temperatura de $765^{\circ} \mathrm{C}$ corresponde à redução da espécie $\mathrm{Mo}^{+6}$ à espécie $\mathrm{Mo}^{+4}$, e na segunda o molibdênio é reduzido para o estado metálico (Mo) em temperatura de $997^{\circ} \mathrm{C}$. 


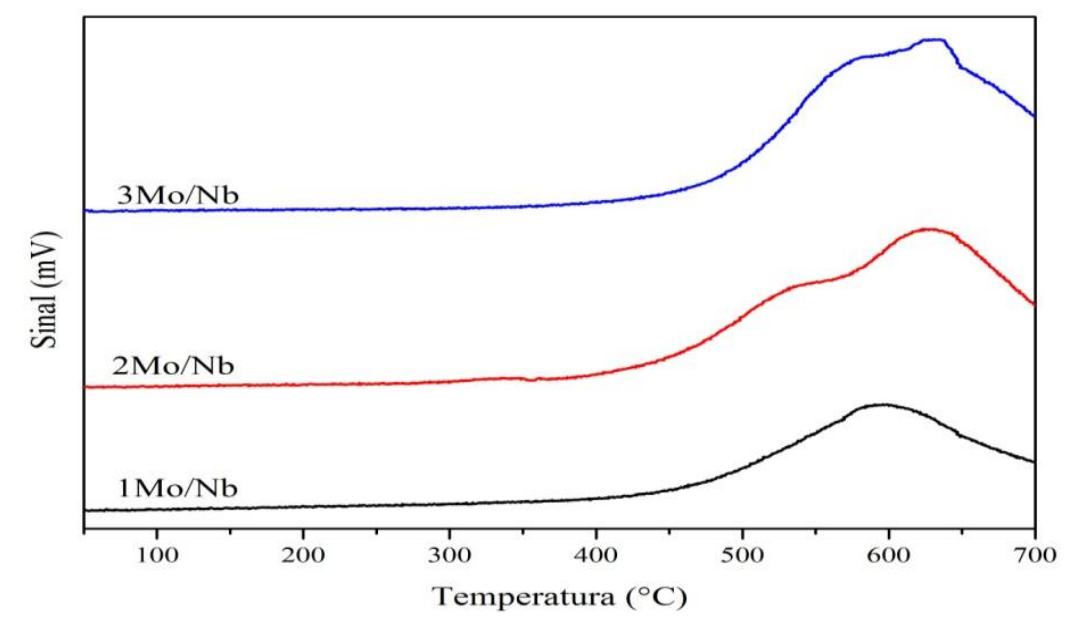

Figura 3 - Perfil de RTP $\left(10^{\circ} \mathrm{C} / \mathrm{min}\right)$ dos catalisadores em $10 \% \mathrm{H}_{2} / \mathrm{N}_{2}, \mathrm{~m}=0,15 \mathrm{~g}$

A Tabela 2 apresenta os resultados de rendimento $\left(\mathrm{R}_{\mathrm{p}}\right)$ à propeno e a $\mathrm{CO}_{2}$ e seletividade $\left(\mathrm{S}_{\mathrm{p}}\right)$ à propeno na temperatura de reação para $10 \%$ de conversão do propano para o suporte $\mathrm{Nb}_{2} \mathrm{O}_{5}$ e catalisadores $1 \mathrm{Mo} / \mathrm{Nb}, 2 \mathrm{Mo} / \mathrm{Nb}$ e $3 \mathrm{Mo} / \mathrm{Nb}$.

Tabela 2 - Resultados da atividade catalítica na ODH para 10\% de conversão do propano.

\begin{tabular}{ccccc}
\hline Amostra & $\mathrm{T}_{\text {Reação }}\left({ }^{\circ} \mathrm{C}\right)$ & $\mathrm{R}_{\mathrm{p}}(\%) \mathrm{C}_{3} \mathrm{H}_{6}$ & $\mathrm{R}_{\mathrm{p}}(\%) \mathrm{CO}_{2}$ & $\mathrm{~S}_{\mathrm{p}}(\%) \mathrm{C}_{3} \mathrm{H}_{6}$ \\
\hline $\mathrm{Nb}_{2} \mathrm{O}_{5}$ & 485 & 9,1 & 0,4 & 84,5 \\
$1 \mathrm{Mo} / \mathrm{Nb}$ & 495 & 8,6 & 0,3 & 55,9 \\
$2 \mathrm{Mo} / \mathrm{Nb}$ & 482 & 8,7 & 0,4 & 50,6 \\
$3 \mathrm{Mo} / \mathrm{Nb}$ & 488 & 9,9 & 0,1 & 83,4 \\
\hline
\end{tabular}

Os resultados da Tabela 2 revelaram que a adição do molibdênio sobre a nióbia não alterou a formação de $\mathrm{CO}_{2}$ nem a temperatura de conversão do propano. A seletividade e o rendimento do $3 \mathrm{Mo} / \mathrm{Nb}$ foram praticamente os mesmos no suporte e diminuíram para $1 \mathrm{Mo} / \mathrm{Nb}$ e $2 \mathrm{Mo} / \mathrm{Nb}$. Os catalisadores $1 \mathrm{Mo} / \mathrm{Nb}$ e $2 \mathrm{Mo} / \mathrm{Nb}$ apresentaram resultados semelhantes, exceto para a temperatura de reação, que foi menor para o $2 \mathrm{Mo} / \mathrm{Nb}$ em aproximadamente $13^{\circ} \mathrm{C}$. O catalisador $3 \mathrm{Mo} / \mathrm{Nb}$ foi mais eficiente por apresentar maior seletividade e rendimento à propeno e menor rendimento à $\mathrm{CO}_{2} \mathrm{em}$ temperatura próxima a menor apresentada na Tabela 2. Neste caso, pode-se concluir que o rendimento e a seletividade à propeno foi crescente com a adição de Mo, sendo máximo na temperatura de $488^{\circ} \mathrm{C}$ para $10 \%$ de conversão do propano.

A Tabela 3 apresenta os valores de conversão do propano $\left(X_{A}\right)$, rendimento à propeno $\left(R_{p}\right)$, seletividade à propeno $\left(\mathrm{S}_{\mathrm{p}}\right)$ e TOF para três temperaturas dos catalisadores $1 \mathrm{Mo} / \mathrm{Nb}, 2 \mathrm{Mo} / \mathrm{Nb}$ e $3 \mathrm{Mo} / \mathrm{Nb}$ e do suporte $\mathrm{Nb}_{2} \mathrm{O}_{5}$ na reação de desidrogenação oxidativa do propano (ODH). De modo geral, a adição crescente do molibdênio não provocou mudanças significativas em relação ao suporte. 
A atividade catalítica do $1 \mathrm{Mo} / \mathrm{Nb}$ começa acima de $500^{\circ} \mathrm{C}$ e com alta seletividade à propeno. $\mathrm{O}$ catalisador $2 \mathrm{Mo} / \mathrm{Nb}$ apresenta alta conversão de propano, indicando maior atividade catalítica, mas a seletividade diminui conforme aumenta a temperatura de reação. $\mathrm{O}$ catalisador $3 \mathrm{Mo} / \mathrm{Nb}$ apresenta comportamento semelhante ao suporte, com alta seletividade à propeno, mas quase não se observa a formação de $\mathrm{CO}_{2}$. No estudo de Meunier et. al. (1997), o catalisador $\mathrm{Mo} / \mathrm{Nb}_{2} \mathrm{O}_{5}$ (5\% p/p Mo), sintetizado a partir de nióbia hidratada e calcinada a $627^{\circ} \mathrm{C}$, apresentou conversão máxima de propano de $15 \%$ a $527^{\circ} \mathrm{C}$, além disso, a atividade catalítica se mostrou muito próxima ao $\mathrm{Mo} / \mathrm{Al}_{2} \mathrm{O}_{3}$, que também foi objeto de estudo de novos catalisadores.

Tabela 3 - Resultados da atividade catalítica na ODH.

\begin{tabular}{ccccccc}
\hline Catalisadores & $\mathrm{T}_{\text {Reaç̃o }}\left({ }^{\circ} \mathrm{C}\right)$ & $\begin{array}{c}\mathrm{X}_{\mathrm{A}}(\%) \\
\mathrm{C}_{3} \mathrm{H}_{8}\end{array}$ & $\mathrm{TOF}\left(\times 10^{-4}\right)$ & $\begin{array}{c}\mathrm{R}_{\mathrm{p}}(\%) \\
\mathrm{C}_{3} \mathrm{H}_{6}\end{array}$ & $\begin{array}{c}\mathrm{S}_{\mathrm{p}}(\%) \\
\mathrm{C}_{3} \mathrm{H}_{6}\end{array}$ & $\begin{array}{c}\mathrm{S}_{\mathrm{p}}(\%) \\
\mathrm{CO}_{2}\end{array}$ \\
\hline \multirow{3}{*}{$\mathrm{Nb}_{2} \mathrm{O}_{5}$} & 486 & 10,7 & - & 9,8 & 91,7 & 3,5 \\
& 498 & 14,7 & - & 13,3 & 90,5 & 2,7 \\
& 510 & 24,1 & - & 20,9 & 86,7 & 0 \\
\hline \multirow{3}{*}{$1 \mathrm{Mo} / \mathrm{Nb}$} & 488 & 0,28 & 0,1 & 0 & 0 & 100 \\
& 500 & 15,4 & 4,2 & 15,0 & 97,5 & 2,5 \\
& 511 & 27,4 & 7,5 & 26,7 & 97,5 & 2,5 \\
\hline \multirow{2}{*}{$2 \mathrm{Mo} / \mathrm{Nb}$} & 487 & 17,1 & 2,4 & 15,7 & 92,0 & 2,3 \\
& 498 & 30,1 & 4,1 & 26,6 & 88,4 & 2,0 \\
& 511 & 70,0 & 9,6 & 43,0 & 61,4 & 5,4 \\
\hline \multirow{3}{*}{$3 \mathrm{Mo} / \mathrm{Nb}$} & 484 & 9,3 & 0,9 & 9,3 & 100 & 0 \\
& 500 & 19,7 & 1,9 & 18,3 & 92,9 & 0 \\
\hline
\end{tabular}

\section{CONCLUSÕES}

O óxido de nióbio se mostrou favorável para ser utilizado como suporte, devido a sua alta temperatura de redução, tornando-o mais estável, e promovendo assim uma forte interação com o molibdênio. A propriedade textural da nióbia foi modificada pela adição crescente de molibdênio, a qual provocou o bloqueio dos microporos pelas espécies de molibdênio superficial. A metodologia empregada na preparação do suporte revelou um material amorfo ao DRX e a adição de $\mathrm{MoO}_{\mathrm{x}}$ não provocou mudanças no perfil do DRX, o que indica que os óxidos estão presentes em um estado amorfo, ou altamente dispersos sobre o suporte. Os resultados de TPR revelaram que a quantidade de molibdênio não alterou significativamente os perfis de redução e observou-se que há presença de picos mais intensos de redução, mas que não correspondem aos picos do molibdênio mássico, o que pode indicar uma interação entre o molibdênio e o nióbio, formando óxidos mistos. Nos resultados da reação de $\mathrm{ODH}$, verificou-se que a presença do $\mathrm{MoO}_{\mathrm{x}}$ sobre $\mathrm{Nb}_{2} \mathrm{O}_{5}$ proporcionou um aumento na 
conversão do propano, sendo mais seletivo o catalisador com menor teor de molibdênio $(1 \mathrm{Mo} / \mathrm{Nb})$, mas para $10 \%$ de conversão o catalisador mais eficiente foi o $3 \mathrm{Mo} / \mathrm{Nb}$ por apresentar maior seletividade e rendimento à propeno em temperatura próxima à menor obtida entre os catalisadores.

\section{AGRADECIMENTOS}

Ao Laboratório de Combustão e Propulsão (LCP) do Instituto Nacional de Pesquisas Espaciais (INPE/Cachoeira Paulista) pelo uso de sua infra-estrutura na síntese e caracterização da nióbia e a FAPESP pela bolsa de Iniciação Científica concedida a Bianca Daniela Costa (Proc. 2012/23064-1).

\section{REFERÊNCIAS}

ARNOLDY, P.; JONGE, J. C. M.; MOULJIN, J. A. Temperature-Programmed Reduction of $\mathrm{MoO}_{3}$ and $\mathrm{MoO}_{2}$. J. Phys. Chem., v. 89, p. 4517-4526, 1985.

CHARY, K. V. R.; RAMESH, K.; VIDYASAGAR, G.; RAO, V. V. Vapour phase alkylation of phenol with methanol over vanadium oxide supported on zirconia. J. Mol. Catal. A: Chem., v.198, p. 195-204, 2003.

CORTEZ, G.G.; FIERRO, J.L.G.; BAÑARES, M.A. Role of potassium on the structure and activity of alumina-supported vanadium oxide catalysts for propane oxidative dehydrogenation Catal. Today, v. 78, p.219-228, 2003.

HERACLEOUS, E.; MACHLI, M.; LEMONIDOU, A. A.; VASALOS, I. A. Oxidative dehydrogenation of ethane and propane over vanadia and molybdena supported catalysts. J. Mol. Catal. A: Chem., v. 232, p.29-39, 2005.

MAMEDOV, E.A.; CORTES-CORBERAN, V. Oxidative Dehydrogenation of lower alkanes on vanadium oxide-based catalysts. The present state of the art and outlooks. Appl. Catal. A., v.127, p. 140, 1995.

MEUNIER, F.C.; YASMEEN, A.; ROSS, J.R.H., Oxidative dehydrogenation of propane over molybdenum-containing catalysts, Catal. Today, v.37, p.33-42, 1997.

PCPDFWIN v. 2.3; Program for Powder Diffraction Files, JCPDS, International Center for Diffraction Data, Pensylvania, USA, 2002 (CD-ROM).

ROQUEIROL, J.; RODRIGUEZ-REINOSO, F.; SING, S.W. in: Characterization of porous solids III, Amsterdam: Elsevier, 1994.

YUAN, L.; BHATT, S.; BEAUCAGE, G.; GULIANTS, V.V.; MAMEDOV, S.; SOMAN, R.S. Novel mesoporous mixed $\mathrm{Nb}-\mathrm{M}(\mathrm{M}=\mathrm{V}$, Mo, and $\mathrm{Sb}$ ) oxides for oxidative dehydrogenation of propane. J. Phys. Chem. B. v. 109, p. 23250-23254, 2005.

ZIOLEK, M. Niobium-containing catalysts - the state of art. Catal Today, v. 78, p. 47-68, 2003 